\title{
CONHECIMENTOS, ATITUDES E CRENÇAS DE HOMENS RESIDENTES EM ARAXÁ- \\ MG QUANTO AO CÂNCER DE PRÓSTATA
}

\author{
Ana Flavia Vieira ${ }^{1}$ \\ Aline do Carmo França Botelho² \\ Recebido em: 09 jan. 2018 \\ Aceito em: 09 dez. 2018
}

\begin{abstract}
RESUMO: O câncer de próstata é o mais incidente entre os homens em todas as regiões brasileiras, e tende a aumentar o número de casos, em resposta ao envelhecimento populacional, pois tem como principal fator de risco a idade do homem. O exame do toque retal, embora eficaz quando combinado com o exame de sangue na detecção precoce das neoplasias da próstata, ainda não é amplamente realizado, pois frequentemente é visto como contrário a masculinidade. O objetivo do estudo foi avaliar conhecimentos, atitudes e crenças de homens acima de 40 anos residentes em Araxá-MG quanto ao câncer de próstata. A pesquisa foi exploratória, quantitativa, realizada através de questionário aplicado para homens acima de 40 anos, na estratégia de saúde da família Vila Estância da cidade de Araxá-MG. Os resultados mostraram que, em geral, os homens entrevistados têm conhecimentos quanto ao câncer de próstata, exames de rastreio e importância do diagnóstico precoce. Entretanto, notou-se que, as ações efetivas, com realização dos dois exames de rotina periodicamente, ainda não são amplamente realidade entre os participantes, pois parcela significativa da amostra nunca realizou os exames preconizados. Ações educativas devem ser intensificadas com intuito de divulgar a importância dos exames de rastreio, bem como, do estilo de vida na prevenção do câncer de próstata.
\end{abstract}

Palavras-chave: Câncer de Próstata. Exame de Toque Retal. PSA.

\section{KNOWLEDGE, ATTITUDES AND BELIEFS OF RESIDENT MEN IN ARAXÁ-MG}

\section{REGARDING PROSTATE CANCER}

ABSTRACT: Prostate cancer is the most frequent among men in all Brazilian regions, and it tends to increase the number of cases, in response to the aging population, since its main risk factor is the age of the man. Rectal examination, although effective when combined with a blood test for the early detection of prostate neoplasms, is still not widely performed because it is often seen as contrary to masculinity. The objective of the study was to evaluate the knowledge, attitudes and beliefs of men over 40 years living in Araxá-MG regarding prostate cancer. The research was exploratory, quantitative, performed through a questionnaire applied to men over 40 years old, in the health strategy of the Vila Estância family of the city of Araxá-MG. The results showed that, in general, the men interviewed had knowledge about prostate cancer, screening tests and the importance of early diagnosis. However, it was noted that the effective actions, with the two routine exams being performed periodically, are not yet widely realized among the participants, since a significant portion of the sample never

\footnotetext{
1 Centro Universitário do Planalto de Araxá (UNIARAXÁ).

${ }^{2}$ Centro Universitário do Planalto de Araxá (UNIARAXÁ). Doutora, UNIARAXÁ. E-mail para correspondência: alinebotelho@uniaraxa.edu.br.
} 
performed the recommended tests. Educational actions should be intensified in order to publicize the importance of screening exams as well as lifestyle in the prevention of prostate cancer.

Keywords: Prostate Cancer. Rectal Touch Exam. PSA.

\section{INTRODUÇÃO}

Sem considerar os tumores de pele não melanoma, o câncer de próstata é o mais incidente entre os homens em todas as regiões brasileiras (INCA, 2016).

O fator de risco mais estabelecido para o desenvolvimento do câncer de próstata é a idade. Aproximadamente $62 \%$ dos casos de câncer da próstata diagnosticados no mundo acometem homens com 65 anos ou mais. Com o crescimento da expectativa de vida mundial, é esperado que o número de casos novos tem um crescimento significativo nos próximos anos. Outro fator importante na etiologia desse tipo de câncer é a dieta. Dietas com base em gordura animal, carne vermelha, embutidos e cálcio têm sido associados ao aumento no risco de desenvolver câncer da próstata. Além disso, também contribui como fator de risco a obesidade, em especial para aquelas neoplasias de comportamento mais agressivo. Em contrapartida, dietas ricas em vegetais, vitaminas D e E, licopeno e Ômega3 aparecem como fatores protetores (SBU, 2008).

No adenocarcinoma da próstata o tempo de duplicação celular é de cerca de 2 a 4 anos, assim o tumor leva em média 15 anos para atingir $1 \mathrm{~cm}^{3}$. Em fases iniciais os adenocarcinomas prostáticos em geral assintomáticos e descobertos devido à elevação do PSA, toque retal alterado (indispensável) ou após tratamento cirúrgico de hiperplasia prostática. Admite-se que o risco de diagnóstico de câncer de próstata durante a vida é de $16,4 \%$ e de óbito, 3,7\% (SBU, 2013).

Segundo Rhoden e Averbeck (2010), a natureza silenciosa do câncer de próstata pode ser exemplificada pelos achados de que a prevalência histológica da neoplasia excede a doença clinicamente manifesta em aproximadamente oito vezes. Os sintomas de disfunção miccional (polaciúria, disúria, redução da força e calibre do jato urinário, noctúria, hesitação, sensação de repleção miccional) são historicamente mencionados como os sintomas mais comumente relacionados ao câncer de próstata.

O diagnóstico do adenocarcinoma de próstata se faz através do estudo histopatológico de espécimes prostáticos, sejam eles obtidos através de biópsia por agulha fina ou através de ressecção transuretral e outros procedimentos prostáticos. $\mathrm{O}$ estudo histopatológico permite a determinação do tipo histológico e grau de diferenciação tumoral, com impacto no prognóstico da doença. A indicação da biópsia transretal se faz a partir da suspeita clínica, em decorrência de alterações no PSA ou toque retal realizado por profissional experiente. Qualquer paciente com uma dessas alterações ou a combinação entre as mesmas deve ser submetido à biópsia prostática (PUNGLIA et al. 2003).

A classificação do câncer de próstata segue o sistema TNM (Tumor, Nódulo e 
Metástase) é a forma mais utilizada para estadiar histologicamente o adenocarcinoma de próstata, trata-se do escore de Gleason. O sistema é graduado de 2 a 10 de acordo com o grau de diferenciação celular, sendo 20 menos agressivo e 10 o mais agressivo (GLEASON; MELLINGER, 1974).

Os pacientes com câncer de próstata de baixo e intermediário risco são aqueles com doença localizada na próstata e baixo PSA. A primeira opção de tratamento para pacientes com expectativa de vida superior a cinco anos e que não tenham contraindicação cirúrgica é a prostatectomia radical. A depender do risco, pode-se optar por realizar, concomitantemente, a linfadenectomia pélvica. Os principais efeitos colaterais da prostatectomia radical, seja ela por via retropúbica aberta, perineal, laparoscópica ou robótica, são a disfunção erétil e a incontinência urinária. Outros tratamentos para doença de baixo risco incluem a radioterapia, hormonioterapia e a vigilância ativa. Já os pacientes de alto e muito alto risco devem receber tratamento mais agressivo, visto o maior potencial metastático da doença nestes casos (GACCl et al., 2008).

Vários fatores socioculturais, incluindo os estereótipos de gênero, crenças e valores que definem o que é ser masculino, têm sido apontados como obstáculos para práticas de cuidado em saúde. Assim o exame do toque retal, embora bastante eficaz quando combinado com o exame de sangue na detecção precoce do tumor de próstata, ainda é relativamente pouco realizado, possivelmente por esbarrar em preconceitos relacionados aos estereótipos de gênero (GOMES et al., 2008).

A Sociedade Brasileira de Urologia (SBU, 2014) recomenda que homens a partir de 50 anos procurem seu urologista para discutir a prática e a realização da avaliação. Aqueles com maior risco da doença (história familiar, raça negra) devem procurar o urologista a partir dos 45 anos, ou até mesmo antes, aos 40 anos. Os exames consistem na dosagem sérica do PSA e no exame digital retal, com periodicidade anual. Esta prática está relacionada à diminuição de cerca de $21 \%$ na mortalidade pela doença em estudos de grande porte e longo seguimento.

A combinação entre os dois exames, tanto o PSA como o de toque retal é a melhor forma de diagnosticar o câncer de próstata o medo da realização do exame do toque retal entre os homens, afeta vários fatores emocionais e físicos por ser um exame de caráter invasivo (PAIVA; MOTTA; GRIEP, 2011).

Segundo Gomes et al. (2008), para os homens, o exame de toque retal não toca apenas a próstata, mas também sua masculinidade, e não pode ser visto apenas como um exame físico, que pode diagnosticar o câncer de próstata precocemente. Diante disso, é necessária uma ampla conscientização e superação de mitos, para que as ações de rastreio precoce dessa neoplasia possa efetivamente obter resultados positivos, os aspectos emocionais e culturais devem ser levados em consideração.

A Sociedade Brasileira de Urologia desenvolve uma iniciativa para promover uma campanha nacional para motivar a população masculina no combate ao câncer de próstata, a campanha já faz parte do calendário nacional de prevenção no Brasil, e tem ganhado 
mais destaque a cada ano, trata-se do "Novembro Azul", uma forma de divulgar a importância da Saúde do Homem, em paralelo a já consagrada campanha do "Outubro Rosa". O Novembro Azul tem diversas atividades em todo o Brasil, como o fórum no Congresso Nacional e no Senado Federal, a iluminação de pontos turísticos, várias palestras informativas e ações educativas em estádios. O objetivo é motivar a população masculina a fazer exames preventivos para o combate à doença (SBU, 2014).

Apesar de sua significativa incidência no Brasil e no mundo, é um tipo de câncer que ainda está muito associado a crenças e medo por parte da população masculina, limitando as possibilidades de diagnóstico precoce, e, consequentemente, de tratamentos mais efetivos. Neste contexto, o presente estudo tornou-se pertinente, pois objetivou avaliar conhecimentos, atitudes e crenças de homens acima de 40 anos residentes em Araxá (MG) quanto ao câncer de próstata.

\section{METODOLOGIA}

Trata-se de um estudo exploratório, transversal e quantitativo, que foi realizado no ESF (Estratégia de Saúde da Família) Vila Estância, no Município de Araxá, MG, com uma proposta de intervenção em relação à prevenção e detecção precoce de câncer de próstata.

Os participantes foram homens cadastrados no ESF Vila Estância em 2015, com idade acima de 40 anos, sendo incluídos todos os que voluntariamente concordaram em participar da pesquisa. A definição dessa idade deve-se ao fato de quando há caso de câncer de próstata na família, homens já com essa idade, devem fazer os exames. Foram excluídos os homens que não tinham condições físicas e psicológicas para responder o questionário.

O instrumento de coleta de dados foi um questionário adaptado para atender a proposta da pesquisa (PAIVA, 2008), com questões gerais, como, idade e escolaridade, e, questões que contemplem os objetivos da pesquisa.

Após coleta de dados houve um trabalho individualizado de conscientização e esclarecimento de questões que os voluntários tiveram, ressaltando a importância das medidas preventivas.

O projeto obedeceu as Diretrizes e Normas Regulamentadoras de Pesquisas Envolvendo Seres Humanos do Conselho Nacional de Saúde (Resolução 466/2012) e incorporou em seu contexto os quatro referenciais da bioética: autonomia, não maleficência, beneficência e justiça. O protocolo de aprovação no comitê de ética do UNIARAXÁ foi 00503/19.

Cada um dos participantes assinou um termo de consentimento livre e esclarecido antes de responder ao questionário. Antes de iniciar a pesquisa foi solicitada a autorização da ESF Vila Estância para a coleta dos dados. 


\section{RESULTADOS E DISCUSSÃO}

A amostra foi composta por 29 homens. De acordo com a tabela 1, a maioria dos voluntários são casados, sendo $62,06 \%$. Quanto à idade, a faixa etária predominante foi de 60 a 69 anos, com $41,37 \%$ dos participantes, seguido pela faixa de 40 a 49 anos, com $31,03 \%$. Em relação à escolaridade, destacou-se o ensino fundamental completo, correspondendo a $44,82 \%$ da amostra.

Os achados do estudo de Paiva, Motta e Griep (2011) são similares aos do presente estudo. Eles tiveram uma amostra com média de idade dos participantes de 61,5 anos, com maioria de homens casados (69,4\%), com baixa escolaridade, sendo que $66,9 \%$ informaram possuir apenas o ensino fundamental incompleto.

Tabela 1. Distribuição dos entrevistados quanto ao estado civil, idade e escolaridade.

\begin{tabular}{lll}
\hline Variável & Classificação & $\mathrm{N}(\%)$ \\
\hline Estado Civil & Solteiro & $4(13,79 \%)$ \\
& Casado & $18(62,06 \%)$ \\
& Divorciado & $3(10,34 \%)$ \\
& Viúvo & $4(13,79 \%)$ \\
\hline Idade & 40 a 49 anos & $9(31,03 \%)$ \\
& 50 a 59 anos & $4(13,79 \%)$ \\
& 60 a 69 anos & $12(41,37 \%)$ \\
& 70 anos ou mais & $4(13,79 \%)$ \\
\hline Escolaridade & Sem escolaridade & $5(17,24 \%)$ \\
& Ensino Fundamental Completo & $13(44,82 \%)$ \\
& Ensino Médio incompleto & $6(20,68 \%)$ \\
& Ensino Médio completo & $2(6,89 \%)$ \\
& Ensino superior incompleto & $1(3,44 \%)$ \\
& Pós-graduação & $2(6,89 \%)$ \\
\hline
\end{tabular}

Fonte: Dados resultantes da pesquisa, 2015.

Os participantes da pesquisa foram questionados quanto a conhecer exame(s) para detecção do câncer de próstata. De acordo com o gráfico 1, 72,41\% responderam afirmativamente e $27,59 \%$ disseram não conhecer nenhum exame. No gráfico 2, quanto aos exames que conhecem, $51,72 \%$ relataram conhecer o exame sanguíneo PSA e 41,37\% disseram conhecer o exame de toque retal.

No estudo de Ascari et al. (2014), realizado em área rural de Santa Catarina, os homens foram questionados também quanto a esses aspectos, e, 61,8\% afirmaram conhecer os exames de detecção do câncer de próstata, $57,3 \%$ disseram ter conhecimento quanto ao exame do PSA, $75 \%$ afirmaram ter conhecimento quanto exame de toque retal. 
Gráfico 1. Distribuição percentual dos entrevistados quanto ao conhecimento de algum exame para a detecção do câncer.

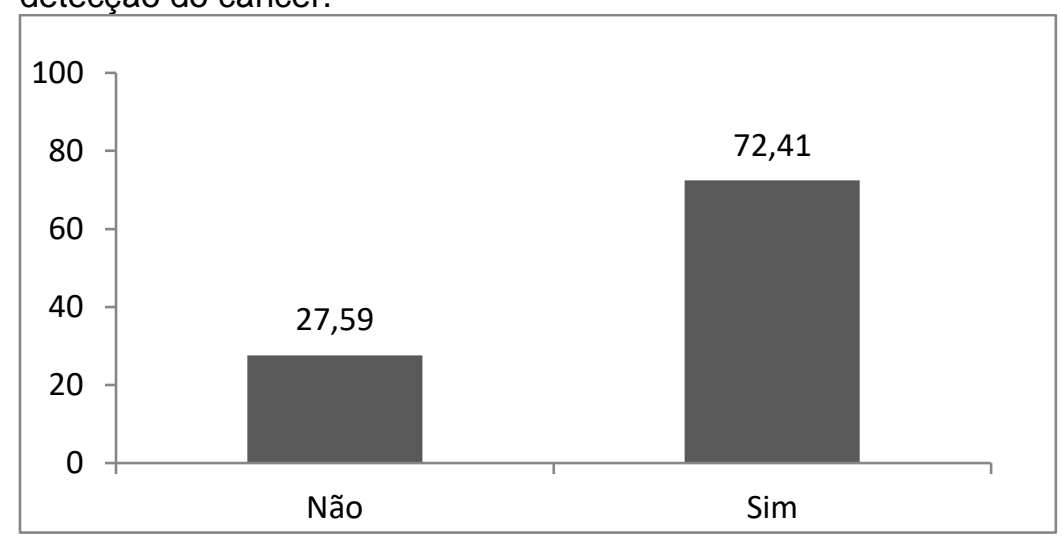

Fonte: Dados resultantes da pesquisa, 2015.

Gráfico 2. Distribuição dos entrevistados quanto aos tipos de exames que conhecem quanto à detecção do câncer de próstata.

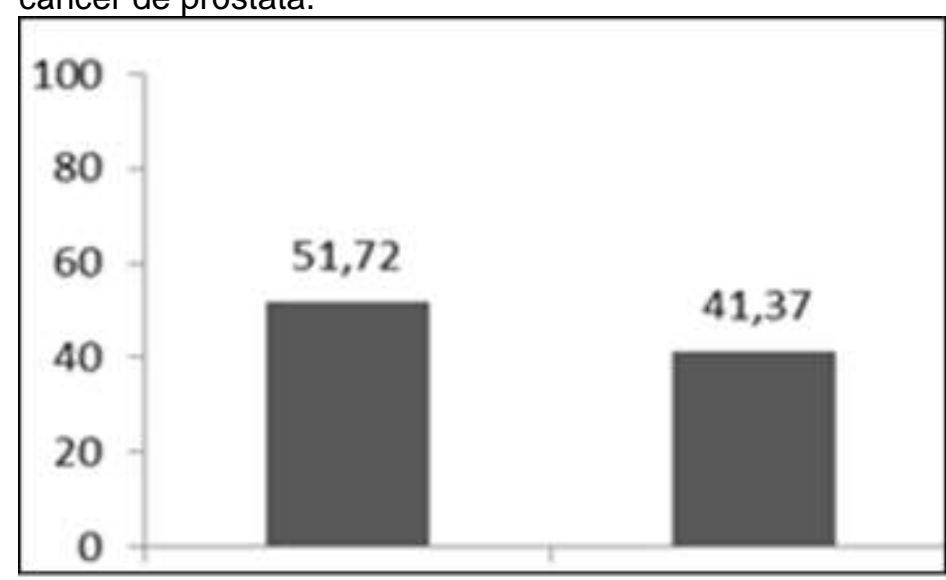

\section{Exame de PSA Exame de Toque}

Fonte: Dados resultantes da pesquisa, 2015.

De acordo com a tabela 2, a maioria dos entrevistados $62,06 \%$, já tinha ouvido falar muito do câncer de próstata, enquanto 37,93\% relataram ter ouvido falar muito pouco do assunto.

Já a tabela 3 refere-se ao meio onde ouviram falar de câncer de próstata, nota-se que $37,93 \%$ foi no PSF, 44,82\% por meio de amigos, 27,58\% através de parentes, pelos meios de comunicação foram 31,03\%, e, outros serviços de saúdes o índice foi de 31,03\%.

Entre os 100 participantes da pesquisa de Vieira et al. (2008) realizada em Fortaleza, 35 usuários afirmaram ter sido orientados sobre câncer de próstata e sua respectiva prevenção; desses, 28 confirmaram que receberam orientações de médicos e um de enfermeiro, e os demais, através de amigos que experienciaram a doença, através da mídia ou agentes de saúde. 
Tabela 2. Distribuição dos voluntários que já ouviram falar de câncer de próstata.

\begin{tabular}{|c|c|}
\hline Variável & $\mathrm{N}(\%)$ \\
\hline Sim, um pouco & $11(37,93 \%)$ \\
\hline Sim, muito & $18(62,06 \%)$ \\
\hline Não & 0 \\
\hline
\end{tabular}

Fonte: Dados resultantes da pesquisa, 2015.

Tabela 3. Distribuição dos entrevistados quanto ao meio que ouviram falar do câncer de próstata.

\begin{tabular}{ll}
\hline \multicolumn{1}{c}{ Variável } & $\mathrm{N}(\%)$ \\
\hline PSF & $11(37,93 \%)$ \\
Amigos & $13(44,82 \%)$ \\
Parentes & $8(27,58 \%)$ \\
TV, rádio, jornal & $9(31,03 \%)$ \\
Outros serviços de saúde & $9(31,03 \%)$ \\
\hline
\end{tabular}

Fonte: Dados resultantes da pesquisa, 2015.

Quando foram perguntados quanto aos hábitos ou situações que poderia aumentar o risco de câncer de próstata, dos entrevistados $72,41 \%$ afirmaram que se tiver caso de câncer de próstata na família aumenta o risco, logo em seguida, com $55,17 \%$, o relato foi que fumar cigarros aumenta o risco. Quanto a ingerir bebida alcóolica e comer alimentos com gordura animal respectivamente, foram referidos 37,93\% e 27,58\% (Gráfico 3).

Segundo Malta et al. (2012), o câncer de próstata está ligado a hereditariedade, sendo que parentes de primeiro grau têm cerca de 8 vezes mais risco, podem se destacar também como fatores de risco: dieta rica em gorduras, ingestão de carnes vermelhas, e baixa ingestão de vitaminas.

Gráfico 3. Distribuição percentual dos entrevistados quanto aos hábitos ou situações que poderia aumentar o risco de câncer de próstata.

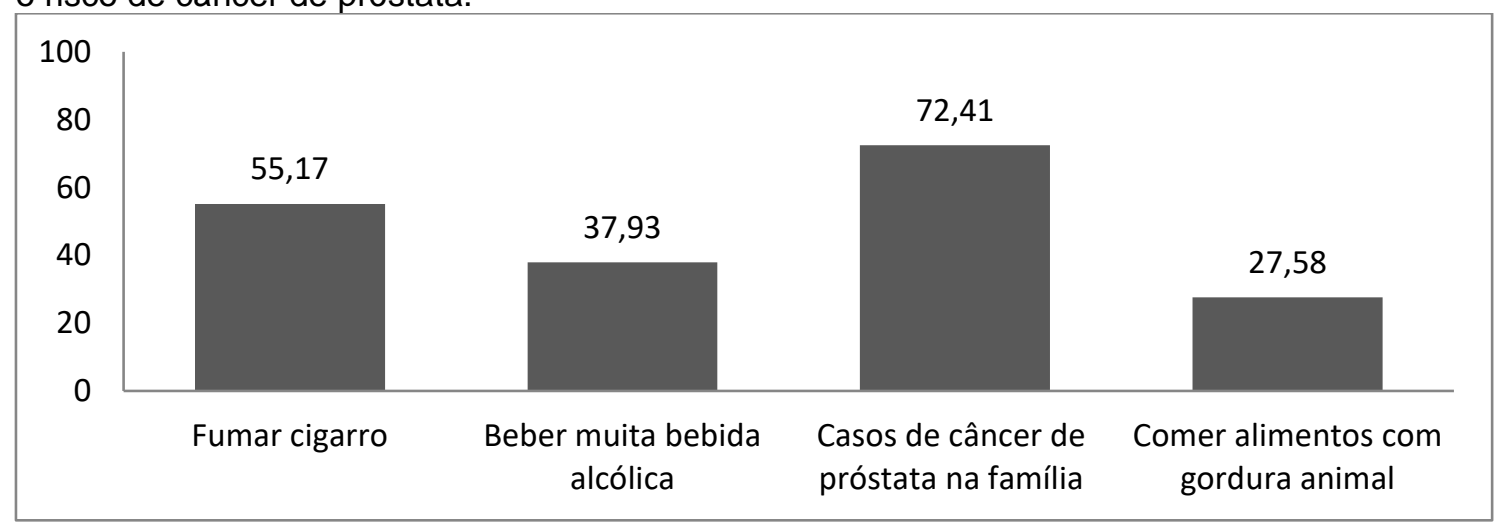

Fonte: Dados resultantes da pesquisa, 2015.

De acordo com a tabela 4, 75\% dos entrevistados relatam que o exame de toque é 
para a prevenção, entretanto, $10 \%$ afirmaram que só realizou o exame quando teve sintomas como dor e ardência para urinar.

No estudo de Paiva, Motta e Griep (2010), 61,3\% voluntários afirmaram que algum médico já havia afirmado que deveria fazer o exame, 54,4\% já realizaram o exame e destes $61,6 \%$ relataram que realiza o exame por rotina e prevenção, apenas $20,9 \%$ relata ser por sintomas e 1,2\% casos de câncer na família.

Tabela 4. Distribuição dos entrevistados com relação ao motivo pelo qual foi realizado o exame de toque

\begin{tabular}{l|l}
\hline Variável & \multicolumn{1}{|c}{$\mathrm{N}(\%)$} \\
\hline Tinha sintomas: dor, ardência para urinar & $2(10 \%)$ \\
Teve casos de câncer de próstata na família & $2(10 \%)$ \\
Somente rotinas de prevenção & $15(75 \%)$ \\
Outros motivos & $1(5 \%)$ \\
\hline
\end{tabular}

Fonte: Dados resultantes da pesquisa, 2015.

Quanto à idade em que o homem deveria começar a fazer o exame de próstata, de acordo com a maioria dos entrevistados do presente estudo $(58,62 \%)$ é a partir de 40 anos (Gráfico 4). Esse resultado foi um pouco menor que o de outro estudo realizado em Juiz de Fora (MG), a grande maioria dos homens (75,6\%) referiu a idade adequada para 0 rastreamento do câncer de próstata é a partir de 40 anos.

Gráfico 4. Distribuição de entrevistados quanto à idade que afirmaram ser a correta para iniciar os exames de próstata.

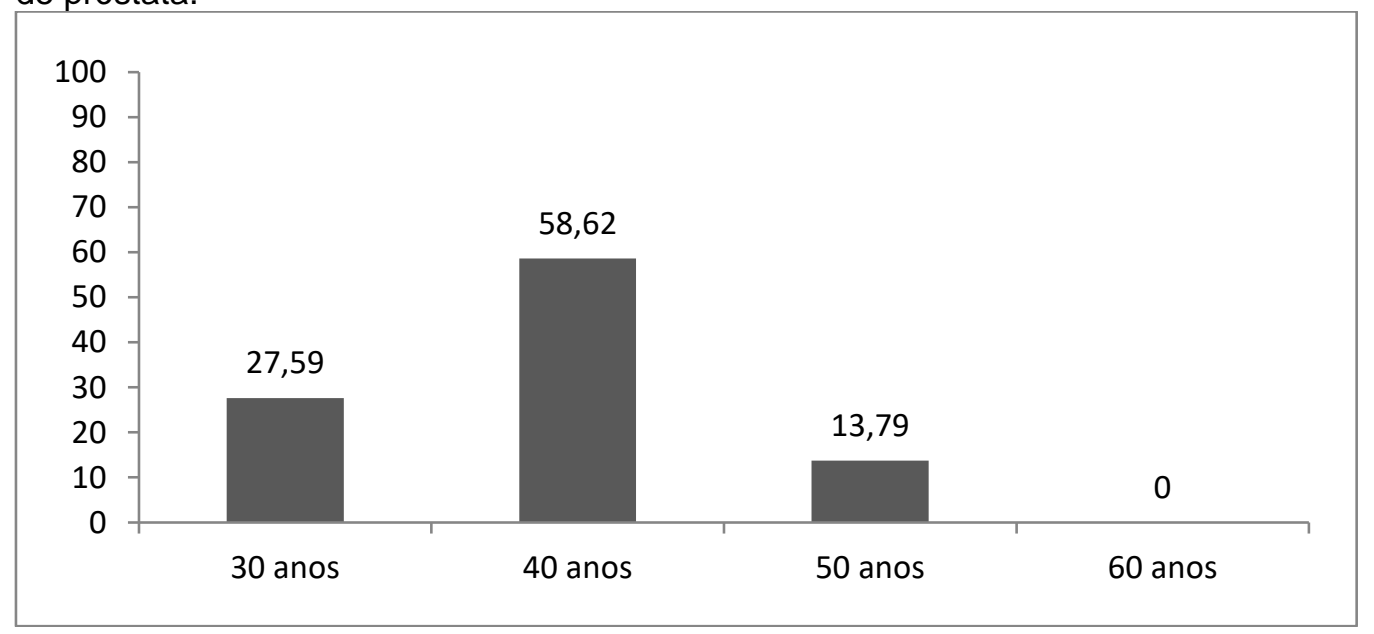

Fonte: Dados resultantes da pesquisa, 2015.

Os homens foram questionados quanto à frequência em que os homens deveriam fazer exames de próstata, a grande maioria, 89,65\% relatou anualmente. E quanto aos exames de próstata apenas para homens que apresentam sintomas urinários, 75,86\% disseram não concordar com isso. E quase totalidade dos voluntários $(93,10 \%)$ afirmou que fazer o exame da próstata é de grande importância para a saúde masculina.

Os participantes foram perguntados se já realizaram alguma vez o exame de próstata (toque), 68,97\% afirmou que já realizou, enquanto 31,03\% relatou nunca ter feito 
o exame (Gráfico 5).

A tabela 5 refere-se a quando foi a última vez que o voluntário realizou o exame de toque, 35\% relatam ter feito a menos de 1 ano, 30\% entre 1 a 2 anos, entretanto $20 \%$ afirmaram entre 3 a 5 anos e apenas 15\% relata não lembrar ou não saber.

No trabalho de Ascari et al. (2014) em Videira (SC), o número de homens que nunca realizaram os exames foi ainda maior, 57,4\%. A pesquisa ainda apontou que a maioria dos homens não realiza anualmente consulta com urologista e destacam a falta de incentivo, por acomodação, por não ter motivo e por não apresentar sintoma.

Gráfico 5. Distribuição dos entrevistados quanto a ter realizado alguma vez o exame de toque.

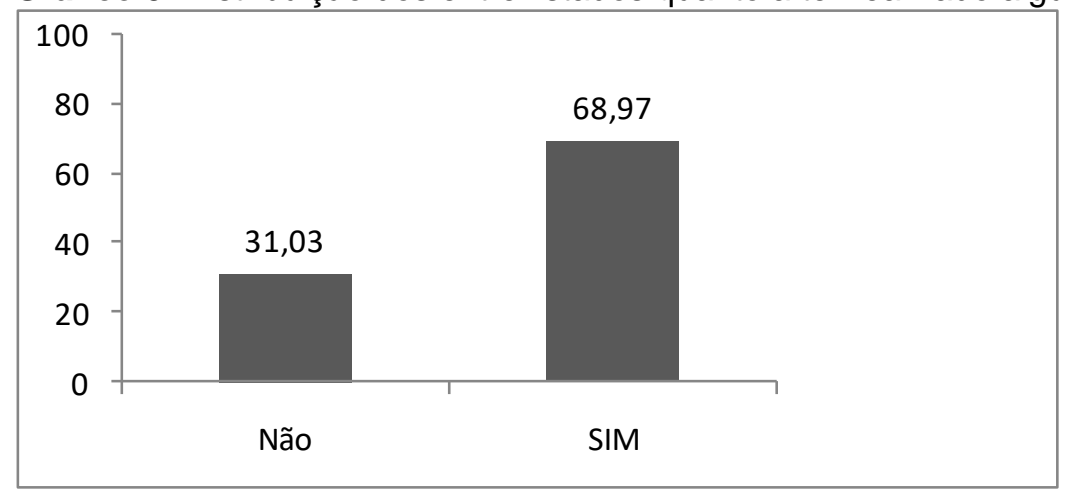

Fonte: Dados resultantes da pesquisa, 2015.

Tabela 5. Distribuição dos entrevistados quanto a última vez em que realizou o exame de toque.

\begin{tabular}{ll} 
Variável & $\mathrm{N}(\%)$ \\
\hline Menos de 1 ano & $7(35 \%)$ \\
Entre 1 e 2 anos & $6(30 \%)$ \\
Entre 3 e 5 anos & $4(20 \%)$ \\
Não sei/não lembro & $3(15 \%)$ \\
\hline
\end{tabular}

Fonte: Dados resultantes da pesquisa, 2015.

É consenso que a melhor forma de detecção precoce do câncer de próstata é associando os dois exames, o toque retal e o PSA. Em geral os homens aceitam mais o exame sanguíneo (PSA) que o exame de toque retal. Diante disso, os homens foram questionados quanto à realização do exame de PSA, 79,31\% afirmaram já ter fez o exame e $20,68 \%$ relatam não ter feito o exame ainda (Gráfico 6). No estudo de Ascari et al. (2014) dos 28 homens que realizaram algum exame, 10 realizaram somente PSA.

De acordo com tabela 6 , quanto a última vez que realizou exame de PSA, 34,48\% afirma que a menos de 1 ano, 24,13\% entre 1 a 2 anos, entretanto 17,24\% relatou entre 3 a 5 anos e 20,68\% afirmaram que nunca fez o exame PSA, apenas 3,44\% não lembra ou não sabe. 
Gráfico 6. Distribuição dos entrevistados quanto a ter realizado alguma vez o PSA.

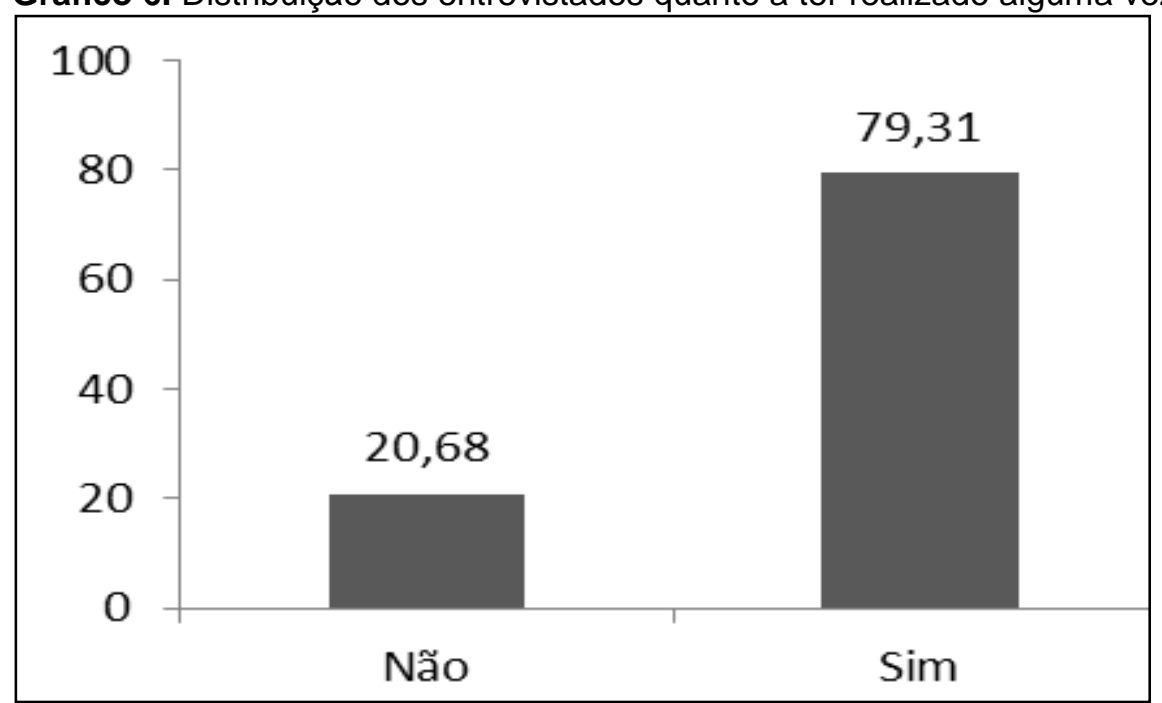

Fonte: Dados resultantes da pesquisa, 2015.

Tabela 6. Distribuição dos entrevistados quanto a última vez que realizou o exame do PSA.

\begin{tabular}{l|l}
\hline Variável & $\mathrm{N}(\%)$ \\
\hline Menos de 1 ano & $10(34,48 \%)$ \\
Entre 1 e 2 anos & $7(24,13 \%)$ \\
Entre 3 e 5 anos & $5(17,24 \%)$ \\
Não sei/ Não lembro & $1(3,44 \%)$ \\
Nunca fez o exame & $6(20,68 \%)$ \\
\hline
\end{tabular}

Fonte: Dados resultantes da pesquisa, 2015.

Quando os entrevistados foram questionados se tem ou teve familiares com câncer de próstata, $82,75 \%$ dizem que não. A maioria também respondeu negativamente quanto a ter algum problema na próstata, com índice de $86,20 \%$.

O conhecimento da verdade e dos mitos referentes ao câncer de próstata pode ser um fator determinante do sucesso ou fracasso das campanhas preventivas. Neste contexto, o questionário também abordou alguns aspectos relativos ao conhecimento sobre o câncer de próstata. Os homens foram também questionados quanto à possibilidade do câncer de próstata ser curado se for detectado precocemente, quanto a esse aspecto, 96,55\% concordaram com afirmativa. Por outro lado os voluntários têm dúvidas quanto a ter uma vida normal em caso de câncer de próstata, sendo que 48,27\% concordaram, 34,48\% relataram discordar, e, 20,68\% não responderam. Em relação à pergunta sobre estar com boa saúde e por isso não ter necessidade de ter a próstata examinada, apenas $24,13 \%$ concorda com afirmativa. Mas quanto ao exame de próstata poder afetar a masculinidade, $27,58 \%$ concorda e $72,41 \%$ relata discordar da afirmativa. 


\section{CONSIDERAÇÕES FINAIS}

Diante dos resultados encontrados é possível afirmar que, de modo geral, os homens entrevistados têm conhecimentos quanto ao câncer de próstata, exames de rastreio e importância do diagnóstico precoce. Entretanto, notou-se que, as ações efetivas, com realização dos dois exames de rotina periodicamente, ainda não são amplamente realidade entre os participantes, pois parcela significativa da amostra nunca realizou os exames preconizados.

Vale salientar ainda que a divulgação por meios de comunicação ainda é pequena, e uma ampliação de campanhas por esses meios poderia ser uma forma de atingir a população masculina de forma mais ampla. Associado a isso, é também necessária uma maior conscientização da relação entre o estilo de vida e o surgimento do câncer, aspectos como tabagismo, dieta e controle do peso precisam ser mais divulgados nas ações educativas voltadas a prevenção do câncer de próstata.

\section{REFERÊNCIAS}

GACCI, M. et al. Predictors of quality of life after radical treatment for prostate cancer. Urologia Internationalis, v. 80, p. 231-6, 2008. Disponível em:

https://www.karger.com/Article/Abstract/127332. Acesso em: 03 dez. 2017.

GLEASON, D. F.; MELLINGER, G. T. Prediction of prognosis for prostatic adenocarcinoma by combined histological grading and clinical staging. The Journal of Urology, v.111, p.58-64, 1974. Disponível em:

http://www.sciencedirect.com/science/article/pii/S0022534717598894?via\%3Dihub.

Acesso em: 03 dez. 2017.

GOMES, R, NASCIMENTO E.F.; REBELLO, L.E.F.S.; ARAÚJO, F.C. As arranhaduras da masculinidade: uma discussão sobre o toque retal como medida de prevenção do câncer prostático. Revista de Ciências e Saúde Coletiva, v.13, p.1975-1984, 2008. Disponível em: https://www.arca.fiocruz.br/handle/icict/246. Acesso em: 05 dez. 2017.

INCA. Instituto Nacional de Câncer. Estimativa 2016. Disponível em: http://www.inca.gov.br/estimativa/2016/sintese-de-resultados-comentarios.asp. Acesso em: 05 dez. 2017.

MALTA, L. R. F. et al. Produção científica da enfermagem em relação ao câncer de próstata: Revisão integrativa. Revista de enfermagem UFPE, v.6, p.3007-3016, 2012. Disponível em:

http://repositorium.sdum.uminho.pt/bitstream/1822/22144/1/Artigo\%20Luciana. Acesso em: 09 dez. 2017.

PAIVA, E. P. Conhecimentos, atitudes e práticas acerca da detecção do câncer de próstata. Tese (Doutorado) UFRJ/EEAN/ Programa de Pós-graduação em Enfermagem, 2008. Disponível em:

http://www.abennacional.org.br/Trabalhos/Elenir_Pereira_de_Paiva.pdf. Acesso em: 09 
dez. 2017.

PAIVA, E. P.; MOTTA, M.C.S.; GRIEP, R.H. Barreiras em relação aos exames de rastreamento do câncer de próstata. Revista Latino Americana de Enfermagem, v.19, 2011. Disponível em: http://www.scielo.br/pdf/rlae/v19n1/pt_11.pdf. Acesso em: 09 dez. 2017.

PUNGLIA, R. S. et al. Effect of verification bias on screening for prostate cancer by measurement of prostate-specific antigen. New England Journal of Medicine, v.349, p.335-42, 2003. Disponível em: http://www.nejm.org/doi/full/10.1056/NEJMoa021659. Acesso em: 09 dez. 2017.

RHODEN, E. L.; AVERBECK, M. A. Cancer de próstata localizado. Revista da AMRIGS, v.54, p.92-99, 2010. Disponível em: http://www.amrigs.com.br/revista/54-01/20488_cancer_de_prostata.pdf. Acesso em: 11 dez. 2017.

SBU. Sociedade Brasileira de Urologia. Alerta para prevenção ao Câncer de Próstata. Rio de Janeiro, p.05-13, jun. 2008. Disponível em:

http://www.sbu.org/indexGeral.php?do=imprensa\&sub=7\&dado_id=195\&site=geral. Acesso em: 11 dez. 2017.

SBU. Sociedade Brasileira de Urologia. Campanha Novembro Azul. 2014. Disponível em: http://www.sbu.org.br/?campanha-novembro-azul-2014. Acesso em: 11 dez. 2017.

SBU. Sociedade Brasileira de Urologia. Sociedade Brasileira de Urologia lança recomendações para o câncer de próstata. 2013. Disponível em: http://www.sbu.org.br/?noticias\&id=1701. Acesso em: 11 dez. 2017.

VIEIRA, L. J. E. S, et al. Prevenção do câncer de próstata na ótica do usuário portador de hipertensão e diabetes. Ciência e Saúde Coletiva, v.13, p.145-152, 2008. Disponível em: http://www.scielo.br/pdf/csc/v13n1/18.pdf. Acesso em: 12 dez. 2017. 